\title{
La forza della realtà nell'agire educativo
}

\section{Michele Pellerey}

Università Salesiana di Roma

doi: 10.7358/ecps-2014-009-pell

pellerey@unisal.it

\section{THE STRENGTH OF REALITY IN THE EDUCATIONAL ACTION}

\section{Abstract}

This paper deals with the role that the impact with reality has in educational action and, in particular, the need to take into account the "face» of the other, especially when he resists our proposals and the educator has to take on the ethical responsibility deriving from such a situation. In this perspective, some of the suggestions of J. Rawls to take into account a reasonableness principle are considered. Then, the position of the "Gestalt" psychologists on the development of cultural, esthetic and moral values, as well as the educational consequences, are described. Finally, on the basis of the theory of capabilities of A. Sen and M. Nussbaum, the educational importance of the conversational theory of G. Pask and D. Laurillard, and the role offeedback, whether positive or negative in the learning process, is considered. The conclusion is that the impact with reality can be a source of new and better perspectives and procedures for educational action.

Keywords: Education, Feedback, Reality, Reasonableness, Values.

\section{INTRODUZIONE}

In una recente pubblicazione Maurizio Ferraris afferma:

Robusto, indipendente, ostinato, il mondo degli oggetti che ci circondano, ma anche dei soggetti con cui interagiamo, non si limita a dire no, a opporre resistenza come per dire "ci sono, sono qui». Con lo stesso gesto con cui resiste, ci offre l'accesso alla massima, e unica, positività a nostra disposizione, allo sfondo tutt'altro che amorfo, ma anzi ricco e strutturato, da cui prendono avvio la sensazione, l'immaginazione, il pensiero, il ricordo, l'attesa, il timore e 
la speranza. E soprattutto dispiega lo spazio delle possibilità (Ferraris, 2013b, p. 9).

Il testo ha sollecitato in me il ricordo di una proposta di P. Meirieu del 1995, quando introduceva il concetto pedagogico di "momento educativo» (Meirieu, 1995). Nel contesto di un'azione diretta alla promozione della crescita personale, sociale, culturale e professionale dei giovani il momento educativo vero e proprio emerge, secondo questo studioso, quando si percepisce la resistenza del bambino o dell'adolescente di fronte ai progetti, agli obiettivi, alla volontà dell'educatore. L'educando sfugge ai propositi e al potere dell'educatore perché non capisce, non accetta, oppure porta in sé altri progetti, altri obiettivi, altra volontà. La riflessione pedagogica si sviluppa proprio quando si decide di non mettere da parte tale resistenza, negandola o sopraffacendola, bensì accettandola e cercando di sviluppare un vero e proprio lavorìo formativo che dirige la propria attenzione e il proprio interesse verso situazioni e soggetti concreti e le loro resistenze. La resistenza dell'altro dal punto di vista educativo non riconduce al potere che potrei esercitare su di lui, ma a quello che devo esercitare su di me. È un rinvio alla mia responsabilità educativa, alla ricerca di un modo di offrire la possibilità di un incontro, al desiderio di comprendere e di aiutare. La manipolazione al contrario si colloca nella volontà ostinata di rimandare sempre all'altro la responsabilità delle difficoltà che si incontrano, nel desiderio di circonvenire per superare l'ostacolo che si frappone.

Di qui ho preso lo spunto per un possibile approfondimento sulla natura dell'azione educativa che deriva dal riconoscimento dell'altro, degli altri, quando questi diventa nostro interlocutore, quando si deve tener conto del "volto dell'altro" e se ne deve assumere la responsabilità etica che ne deriva (Lévinas, 1990). Cercherò di sviluppare tale proposito mediante alcuni passaggi successivi. In primo luogo esplorerò l'apporto che a partire da alcune suggestioni provenienti da J. Rawls può derivare dalla considerazione di quello che nell'ambito dell'amministrazione della giustizia è stato definito il canone o principio di ragionevolezza. In secondo luogo evocherò la posizione degli psicologi della Gestalt in merito alla generazione dei valori estetici, culturali e morali e alle conseguenze educative che ne derivano. Quindi sarà utile al nostro assunto presentare brevemente il contributo educativo che proviene dalla teoria delle capabilities di A. Senn e M. Nussbaum. Su questa base concettuale emerge l'importanza di valorizzare in ambito pedagogico la prospettiva conversazionale avviata da G. Pask e ripresa di D. Laurillard e il ruolo che il feedback sia negativo, sia positivo, sia intrinseco, sia estrinseco, può avere nello sviluppo di tale approccio 


\section{VERSO UN PRINCIPIO \\ O CANONE DI RAGIONEVOLEZZA PEDAGOGICA}

La forza che l'altro, nel nostro caso l'educando o il gruppo degli educandi, può esercitare sull'agente, nel nostro caso l'educatore, sembra essere presa in considerazione più in generale da J. Rawls, quando distingue tra razionalità e ragionevolezza nell'azione (Rawls, 2006 e 2008). In particolare trattando del liberalismo politico egli ha indicato, secondo Maffettone, una definizione chiara di persone ragionevoli. Esse, infatti, «sono disposte a regolare la propria condotta in base a principi che tengono conto anche degli altri. In questo modo le persone ragionevoli rendono possibile la cooperazione in termini equi e ciò consente una giustificazione reciproca delle norme di comportamento» (Maffettone, 2010, p. 103). Se, invece, non si tiene conto degli altri, ma solo della coerenza del proprio ragionamento o del ragionamento in sé, si persegue una modalità definita "razionale». "Quello che manca agli agenti in quanto razionali è la sensibilità che consente di entrare in una cooperazione equa. Rawls dice addirittura che un agente puramente razionale assomiglierebbe a uno psicopatico» (ivi, pp. 103-104) ${ }^{1}$. Un principio di ragionevolezza, insieme a uno di equità, viene evocato, dunque, nell'esplorare il terreno non solo della giustizia, ma soprattutto della convivenza democratica.

Non è il caso di esplorare lo sviluppo che nel diritto sia costituzionale, sia penale, sia civile, a livello europeo, ma anche italiano, ha avuto la considerazione di un principio di ragionevolezza. La letteratura in proposito è assai vasta $^{2}$. La Corte Costituzionale Italiana ha introdotto quello che è stato definito "canone o principio di ragionevolezza», un principio da adottare nella valutazione della coerenza delle leggi rispetto alla Costituzione. Ne è nato un dibattito interessante che ha portato alcuni esperti a richiedere precisazioni concettuali e norme procedurali e non solo il riferimento a casi specifici ${ }^{3}$. Tuttavia, a mio avviso, questa discussione richiederebbe l'utilizzo, come vedremo in seguito, di un apporto concettuale oggetto attualmente di notevole interesse nella letteratura internazionale, quello di saggezza pratica.

A.M. Passaseo (2012) ha esplorato le conseguenze della dottrina di Rawls sul piano dei processi educativi diretti alla formazione del cittadino. Tali processi dovrebbero essere orientati a promuovere due fondamenta-

1 La citazione è tratta da Rawls, 1993, p. 39.

2 Un testo fondamentale di riferimento è Bongiovanni, Sartor, \& Valentini, 2009. Contributi significativi sono stati offerti da: Sandulli, 1975; Zagrebelsky, 1983; Hickeman, 2004; La Torre \& Spadaro, 2007.

3 Se ne può avere un'idea esplorando la rete Internet usando l'espressione «Principio di ragionevolezza e Corte Costituzionale». 
li competenze umane: la razionalità e la ragionevolezza. La prima riguarda la capacità del cittadino di concepire il bene cioè il «suo essere in grado di effettuare una scelta razionale tra fini di varia natura (filosofica, religiosa o morale). Tale scelta può essere definita responsabile quando a determinarla intervengono tutte quelle facoltà intellettive utili a che essa non sia né arbitraria né imposta dall'esterno, ma venga sottoposta a quelle procedure di controllo che consentano al soggetto la valutazione e la 'presa in carico' della scelta stessa. Pertanto è sulla coltivazione della capacità di scegliere che deve concentrarsi l'azione educativa che miri al raggiungimento dell'uso della razionalità». L'autrice quindi esamina la natura di tale capacità di scelta e le azioni educative che possono essere messe in atto per promuoverla.

La seconda competenza è specificatamente orientata a sollecitare la capacità di ragionevolezza. Per approfondire questa prospettiva la Passaseo ricorre ad alcune citazioni. Le prime due sono del filosofo del diritto F. Viola:

Sembra che il pluralismo del nostro tempo non possa che essere governato dalla ragionevolezza [...]. Le società politiche hanno perso la compattezza della loro cultura di fondo e, conseguentemente, il consenso sociale intorno a dei valori condivisi e, soprattutto, intorno al modo di interpretarli è in decomposizione. In queste condizioni il rischio che i giudizi di ragionevolezza siano arbitrari cresce. Eppure al contempo si rafforza la convinzione che solo con la ragionevolezza si potrà affrontare il pluralismo e che si tratta di una via obbligata. (Viola, 2003, p. 163)

E più avanti:

Proprio intorno al valore della ragionevolezza si sta registrando un incontro, che forse aspira ad essere qualcosa di più di un consenso per intersezione, tra le differenti sfere della vita pratica. Si va edificando un modello utopico di uomo ragionevole, di cittadino della società globale, equipaggiato per navigare nel mercato delle idee e per risolvere i conflitti culturali. (ivi, p. 164)

A supporto ulteriore di tale impostazione viene ripreso un testo di G. Zagrebelsky:

$[\ldots]$ «ragionevole» $[\ldots]$ è colui che si rende conto della necessità, in vista della coesistenza, di addivenire a "composizioni» in cui vi sia posto non per una sola, ma per tante «ragioni». Non l'assolutismo di una sola ragione e nemmeno il relativismo rispetto alle tante ragioni (una o l'altra, pari sono), ma il pluralismo (le une e le altre, per quanto possibile, insieme). (Zagrebelsky, 1992, p. 203)

In definitiva emerge come caratterizzazione fondamentale del processo educativo un'azione educativa da parte dell'educatore, che sia ragionevole, nel senso che riconosca le ragioni dell'altro, in questo caso l'educando, e cerchi 
di rielaborare i suoi progetti di intervento tenendo conto del suo "volto». Tale comportamento sta anche alla base della finalizzazione della sua azione verso la promozione di un capacità di ragionevolezza da parte dell'educando: da una parte, infatti, egli si costituisce come modello di agente ragionevole; dall'altra, guida l'educando nell'esercizio della capacità di usare la ragione in termini di ragionevolezza. Alla base di tale competenza, nell'educatore, come nell'educando, sta la capacità di distinguere, secondo Rawls, gli individui ragionevoli dagli irragionevoli. I primi: «[...] pronti a proporre, o ad accettare quando sono altri a proporli, i principi indispensabili a specificare termini di cooperazione che tutti possano considerare equi» e ad accettare «di dover onorare questi principi anche contro i propri interessi (se le circostanze lo richiedono), purché ci si possa aspettare che anche gli altri li onorino». Mentre si riconosce che «è irragionevole non essere disposti a proporre principi di questo tipo o non onorare termini di cooperazione equi quando ci si può ragionevolmente aspettare che gli altri li accettino» o peggio «fingere di proporli o di onorarli ma essendo pronti, se arriva l'occasione, a violarli a proprio vantaggio" (Rawls, 2006, pp. 9-10).

La Passaseo così riassume la sua proposta:

La capacità di giungere ad un accordo tra molti - di pervenire, per dirla con Rawls, ad un consenso per intersezione - si fonda sul potere morale del senso di giustizia: esso è attitudine ad utilizzare la ragione in termini di ragionevolezza. E richiesto ai soggetti, dunque, il padroneggiamento di due differenti modi di ragionamento: il primo si costruisce all'interno di se stessi, al fine di arrivare a determinare una propria coscienza morale, religiosa, filosofica; il secondo si costruisce insieme ad altri, al fine di giungere ad una decisione condivisa.

\section{PRINCIPIO DI RAGIONEVOLEZZA E SAGGEZZA PRATICA}

La posizione di Rawls, e in genere dei giuristi che valorizzano il principio di ragionevolezza, distinguendo tra processi razionali e processi di ragionevolezza, può essere facilmente collegata alle distinzione aristotelica tra razionalità teorica e razionalità pratica. L'espressione «ragionevolezza» ha come sinonimi in italiano "buon senso», «equilibrio», «legittimità», «saggezza» nell'agire. Essa potrebbe favorire un'interpretazione riduttiva, quasi intendendola come una forma attenuata di logica rigorosa, un cedimento verso un razionalità poco seria, o, almeno, una forma di pensiero debole. Una posizione a mio avviso poco fondata sia teoricamente, sia operativamente. Aristotele descrive le competenze nel pensare a partire dall'enunciazione di cinque virtù dianoetiche o intellettuali. In un linguaggio e prospettiva odierni esse si potrebbero 
così descrivere: la scienza (epistéme), competenza nel promuovere la propria conoscenza e nell'organizzarla attraverso la riflessione e il ragionamento (intelligenza discorsiva); la sapienza (sophia), competenza nel ricercare i principi di riferimento e nel dare senso e valore alle proprie conoscenze e alle vicende umane; l'arte (tèchne), competenza tecnico-pratica nel progettare, realizzare e utilizzare gli artefatti umani; la saggezza pratica (phrònesis), o prudenza, competenza nel decidere come agire e come attuare quanto deciso; l'intelligenza (noùs), competenza nel capire, nel cogliere il significato, nel concettualizzare l'esperienza (intelligenza intuitiva).

Due di queste competenze rientrano in quella che viene denominata "ragion pratica», il pensiero che guida e sostiene l'azione, si tratta dell'arte e della saggezza pratica ${ }^{4}$. Ma sarebbe riduttivo comprendere le altre in una sorta di «ragion teoretica», staccata dall'identità anche operativa del soggetto, in quanto esse caratterizzano da molti punti di vista la persona umana in qualità che incidono profondamente sulla maniera di vedere, interpretare, giudicare e agire nella realtà in generale e in quella educativa in particolare (Berti, 1987). In altre parole la distinzione proposta sul piano dell'analisi dei processi di pensiero deve portare sul piano del realtà personale, e quindi sul piano educativo, a una loro integrazione dinamica. D'altra parte, tali processi non si metterebbero in moto e non si evocherebbero reciprocamente, se non intervenissero forze motivazionali adeguate non solo per attivare, ma anche per dirigere e sostenere l'azione umana ${ }^{5}$.

Un catalogo di questo tipo può aiutare a cogliere alcune dominanti presenti nella maniera di procedere degli educatori. In generale occorre subito osservare come il mettere il pensiero al servizio dell'azione, il sapere come quadro di riferimento per l'agire, possa anche portare verso due direzioni pericolose. Da una parte un certo deduttivismo ideologizzante, che vuol racchiudere la complessità del reale in categorie precostituite e dedurre norme per l'azione da quadri teorici astratti; dall'altra, il rimanere prigionieri delle

4 In questi ultimi anni sono apparsi molti studi, soprattutto in ambito culturale anglofono, sul ruolo della saggezza pratica in generale e nel contesto educativo in particolare. Si possono citare ad esempio: Sternberg \& Jordan, 2005; Ferrari \& Potworowski, 2008; Hall, 2011; Schwartz, 2011.

5 Accanto a queste competenze nel pensare oggi spesso si evocano altre forme di razionalità, alcune già considerate nella tradizione filosofica antica, altre segnalate più di recente: la competenza argomentativa, che mira a conseguire non tanto certezze, quanto un'adeguata plausibilità alle proprie conclusioni; la competenza retorica che è rivolta a persuadere gli altri, cercando non tanto di modificare i loro comportamenti, quanto le loro convinzioni. E poi, le forme di pensiero che guidano l'interpretazione dei testi e delle vicende umane, l'ermeneutica; la considerazione della soggettiva percezione del senso e significato delle vicende esistenziali, come la fenomenologia. 
immediate esigenze delle situazioni, con una certa tendenza all'esser più faccendieri, che seri operatori. E qui sta il valore di una ragione bene educata, ponte e mediazione tra quanto è stato elaborato sul piano dell'interpretazione e della riflessione critica, individualmente e collettivamente, e quanto le circostanze, i tempi e i luoghi pongono come sfida e sollecitazione alla capacità di progetto e d'intervento.

A tale fine è utile approfondire la dinamica propria del ragionamento che sta alla base delle decisioni di ordine pratico. Essa si basa su due caposaldi: il fine che si vuole raggiungere e la conoscenza approfondita della condizioni nelle quali occorre agire. Oggi si insiste sull'importanza di formare i giovani a una cittadinanza attiva e partecipativa, ma l'identificazione delle qualità di tale forma di cittadinanza nel contesto di un mondo che ci avvolge in cerchi concentrici dal luogo natale, alla nazione, all'Europa, al mondo, pone non piccoli problemi di comprensione delle esigenze di tali molteplici appartenenze e di capacità di rispondere a esse in maniera autonoma e responsabile. Essere onesti in tale contesto implica saper gestire se stessi nel complesso delle relazioni e degli impegni di tipo lavorativo, famigliare, sociale e politico, in un sistema soggetto a rapidi cambiamenti e a crisi ricorrenti. Ma, se anche sono chiare le finalità ideali che sollecitano all'azione, occorre conoscere e interpretare con perspicacia le condizioni concrete della domanda di intervento, rispetto alle quali scegliere le forme e le modalità d'intervento e di relazione.

In questo contesto, la questione fondamentale dell'identità e della qualità del ragionamento proprio della saggezza pratica non sta tanto nella chiara visione ideale che sollecita l'azione, quanto nella conoscenza della situazione nella quale occorre intervenire, con le sue esigenze, i suoi condizionamenti e le sue opportunità. Come già accennato forme di razionalismo ideologizzanti sono presenti sia in varie teorie pedagogiche, sia in ambito pratico educativo. Accettare i condizionamenti che provengono dalla realtà non è sempre agevole, né gratificante. Ma è anche deleterio rimanere prigionieri di situazioni difficili, che vengono considerate senza speranza. In questa complesso bilanciamento tra idealità e realtà si evidenzia la qualità personale di chi ha sviluppato la capacità di prendere decisioni prudenti e responsabili attraverso un diuturno esercizio.

\section{SAgGEZZA PRATICA E INTUIZIONE EDUCATIVA}

I grandi educatori hanno saputo confrontare le decisioni prese con le loro conseguenze proprio sul piano della loro fecondità in ordine al bene che si intende promuovere e affinare la propria capacità di cogliere nella sua totalità e complessità le situazioni nelle quali doveva intervenire. Questa capacità 
è propria dell'intelligenza intuitiva. Purtroppo nella storia della riflessione critica sulla natura della ragione si è persa di vista nel tempo la natura e l'importanza di quest'ulteriore qualità cognitiva. $\mathrm{Ci}$ si concentrati sulla dimensione cosiddetta discorsiva della ragione, quella che presiede l'analisi critica analitica delle questioni e delle situazioni, perdendo molte volte di vista la sua dimensione intuitiva, quella che ci permette di coglierne la totalità e il significato. Oggi si tende a rileggerla e rivalutarla nella sua importanza da molti punti di vista, anche sul piano scientifico-matematico. Massimo Baldacci ha evocato recentemente tali due dimensioni, definendole come momento intuitivo e momento razionale della cognizione nel quadro di un insieme di antinomie educative (Baldacci, 2012, pp. 314-318) ${ }^{6}$.

Certamente il processo intuitivo per la sua immediatezza sembra talvolta essere guidato da forme impulsive segnate dall'emotività. Ma lo studio sullo sviluppo delle competenze di persone esperte nei loro ambiti professionali ha messo in luce quanto già a suo tempo aveva prospettato Aristotele. Il soggetto esperto riesce a cogliere agevolmente il quadro completo e articolato delle situazioni da affrontare e di conseguenza a decidere e ad agire in modo fluido, appropriato e senza sforzo. Un apporto alla comprensione della dinamica psicologica che sta alla base di tali comportamenti viene dalle ricerche della Gestalt sul ruolo delle capacità intuitive degli esperti solutori di problemi. Questi sono sensibili alle sollecitazioni provenienti dalla configurazione degli elementi informativi e di fatto e pronti a cogliere quella che è stata definita una "buona forma», cioè una configurazione coerente e ben strutturata che non solo permette di comprendere la questione e la situazione in oggetto, ma anche una sollecitazione a intervenire in essa in modo appropriato ed efficace. Prevale decisamente l'intuizione o insight, che riesce a intus legere a "leggere dentro» le situazioni in maniera agevole e pertinente ${ }^{7}$. Torneremo nel prossimo paragrafo su questo apporto più in dettaglio.

Questo ruolo dell'intuizione, come già accennato, è stato contrapposto spesso alla riflessione, al ragionamento discorsivo, al controllo critico. In realtà occorre trovare un equilibrio tra le due componenti del pensiero,

6 M. Baldacci nel quadro della suo criticismo moderato considera varie antinomie educative che inevitabilmente sollecitano il giudizio pratico dell'educatore (Baldacci, 2012, pp. 314-318).

7 E la tesi sostenuta da molti psicologi della Gestalt, che hanno evidenziato il ruolo centrale dell'insight nella soluzione di problemi anche esistenziali, nella comprensione profonda e nel dare senso e significato alle situazioni, rispondendo quasi a un appello che ci proviene dal mondo esterno (in molti casi anche dal mondo interno), perché ci apriamo alla cosiddetta «buona forma» o totalità significativa. Ad esempio M. Wertheimer lo ha descritto nel processo di soluzione di problemi (Wertheimer, 1965). Una trattazione più approfondita la si può trovare in Fuller, 1990. 
perché da una parte c’è il pericolo di muoversi impulsivamente, dall'altra rimanere a ruminare indefinitamente prima di decidersi ad agire (Gollwitzer, 1990). Una verifica esterna in genere è data o da prove empiriche, o dal confronto con altri; cioè la componente intuitiva o soggettiva deve confrontarsi spesso con aspetti più oggettivi e più intersoggettivi. Oggi si tende a evocare il ruolo della ricerca che in ambito educativo dovrebbe aiutare a conoscere e interpretare le condizioni nelle quali occorre prendere le decisioni, che siano il più possibile coerenti ed efficaci rispetto al quadro delle finalità che si intendono perseguire. Queste devono rispondere poi a una adeguato percorso di progettazione. D'altra parte la ricerca empirica, la ricerca cioè diretta a conoscere le situazioni di fatto, anche quando è di natura operativa, cioè orientata a impostare un'azione educativa rispondente all'emergere dei bisogni riscontrati, può essere descritta a partire da due sue polarità fondamentali: distale e prossimale, tenendo conto della distanza a cui ci si colloca rispetto alla situazione specifica (Pellerey, 2013). Un esempio di ricerca di natura distale è una ricerca basata sulla rilevazione di numerose informazioni che vengono trasformate in dati ed elaborate statisticamente. Viene evocata in questo caso una razionalità di tipo scientifico. Tali ricerche forniscono informazioni di tendenza. Ricerche di natura prossimale sono per lo più di natura fenomenologica e spesso evidenziano lo stato soggettivo di singole persone. Ambedue le metodologie (distale e prossimale) forniscono elementi di riferimento per prendere decisioni: la prima più in generale in quanto rivolta a un gruppo più o meno numeroso, la secondo più specifica e rivolta a situazioni personali. Forse gli sviluppi della pedagogia sperimentale hanno fatto trascurare proprio quella fonte di conoscenza che ha caratterizzato molti grandi educatori, quell' «intelligenza educativa», collegata alla competenza intellettuale di natura intuitiva che consente di cogliere l'appello che una comprensione profonda a globale dello stato di fatto rivolge all'educatore.

\section{IL RUOLO DELLA REALTÀ OGGETTIVA NELL'ORIGINE DEI VALORI SECONDO GLI PSICOLOGI DELLA «GESTALT»}

Un contributo poco conosciuto è quello che proviene da alcuni dei più eminenti fondatori della psicologia della forma o della Gestalt, in particolare da W. Koehler (1938, 1947, 1967, 1971) e M. Wertheimer (1961 e 1967). Essi avevano sviluppato una critica serrata delle teorie che ipotizzavano un'origine puramente soggettiva dei valori, sia estetici, sia culturali, sia morali. Secondo tali psicologi questi non emergono sulla base di spinte o pulsioni irrazionali, ma neppure da assegnazioni di valore arbitrariamente date a cose in se stesse 
indifferenti. Un valore non deriva da un giudizio soggettivo e arbitrario. Un atto in sé ingiusto non diventa giusto per la preferenza che gli viene attribuita da un soggetto. Una sentenza che in sé, in uno specifico contesto, è ingiusta, non può mutare il suo carattere per la valutazione positiva soggettiva di un giudice. C'è una forza inerente alle cose, viste nella loro totalità e contestualità; c'è un'esigenza interna alle configurazioni della realtà oggettiva, che non può essere superata da soggettive, private, considerazioni di valore.

Questa presa di posizione decisa, che si oppone a tanta psicologia del tempo (siamo negli anni Trenta e Quaranta), deriva dai caratteri propri che contraddistinguono la psicologia della forma. Nella sua più fondamentale impostazione questa teoria psicologica coglie l'origine dei valori nella percezione di una totalità di significato e nelle sue esigenze. È una posizione che si riallaccia al concetto di Gestalt e alle leggi che ne governano la realtà (in particolare la legge della "buona forma» o legge della "pregnanza»). La percezione di una buona forma, la soluzione di un problema, nascono da una necessità interna alla struttura stessa degli oggetti percepiti nel loro contesto, dalle esigenze interne delle relazioni tra gli elementi che costituiscono lo spazio del problema. Analogo principio deve reggere anche nel campo dei valori.

Certo il soggetto non è passivo in tutto questo, egli esplora, riflette, controlla, ma la percezione di una totalità significativa, di una soluzione valida non è un fatto puramente soggettivo, come spesso si è affermato, bensì ha radici nella realtà stessa degli oggetti, delle azioni, delle relazioni, colti nel loro contesto, nella loro collocazione nel tempo e nello spazio. All'interno delle situazioni di fatto, viste nel loro complesso, emergono spinte e sollecitazioni, che seguono leggi di totalità e che orientano e sostengono il soggetto nel giungere a riconoscere significati e valori. Il punto sta in questo: esistono contesti concreti e circoscritti, la struttura generale dei quali trova una affinità in esigenze soggettive, ma la sollecitazione (il «vettore» direbbe Koehler) non ha la sua origine nell'io (Koehler, 1969, p. 68), bensì fuori di esso. In tutto ciò gioca un ruolo decisivo il concetto di requiredness. I valori, ad esempio, sono definiti come totalità di significato positivamente o negativamente richieste in quel preciso punto e contesto del mondo vitale. Il significante esterno che appella e sollecita il soggetto costituisce un luogo che implica necessariamente un significante interno: una necessità interna che emerge nel contesto di una necessità esterna. Tale requiredness non è una spinta o pulsione irrazionale, bensì costituisce come un appello razionale che si colloca alla radice delle nostre motivazioni ${ }^{8}$. E qui sta il carattere di appello

${ }^{8}$ Ad esempio, M. Wertheimer nel descrivere le forze interne alla situazione problematica che sollecitano verso una soluzione dice: «Quando il problema è compreso, SI [la situazione iniziale] contiene tensioni strutturali che vengono eliminate in S2 [la situazione finale]. La tesi 
o sollecitazione del valore. Infatti esso è una totalità di significato che nel momento stesso in cui si costituisce esercita una richiesta di accettazione o rifiuto, esercita cioè una spinta, o tensione, secondo una direzione precisa.

L'esperienza del valore è dunque nel suo stesso nascere una esperienza di doverosa accettazione di un «dover essere» (positive requiredness), oppure di doverosa accettazione di un "non dover essere» (negative requiredness). La psicologia, secondo Koehler, deve capire che la quintessenza dell'umana esperienza è l'esperienza di un appello (the experience of demand; Koehler, 1971, p. 193). Questa richiesta non deriva da una valutazione soggettiva, cioè non siamo noi a dare un valore alle cose o alle azioni, bensì dalla struttura stessa della totalità significativa. "I valori non emergono dagli atteggiamenti soggettivi del singolo» (ivi, 362), essi «sono collocati negli oggetti e nelle occorrenze» (ivi, 364). I valori sono esigenze che emergono in un contesto specifico, e non idee che balzano fuori nella nostra testa, considerato nelle sue relazioni con la totalità del mondo vitale implicato. In particolare un valore positivo può essere sollecitato da un'azione che ci sentiamo in obbligo di fare. Si ha una pressione morale su di noi e non un'emanazione da un nostro stato d'animo. Non sono bisogni derivati solo dal nostro interno, ma esigenze strutturali poste dai significati stessi.

Questa impostazione viene applicata sia a valori estetici, conoscitivi e culturali, sia a valori morali. Nel caso di questi ultimi occorre partire dalla considerazione della struttura della situazione, in cui una certa azione può presentare se stessa come una cosa da fare giusta o sbagliata; un'azione già eseguita può mostrare se stessa come da condannare o da approvare. Le cose che «debbono essere fatte» e i fatti che «debbono essere approvati» sono valori morali positivi. Le cose che «debbono essere evitate», i fatti che «debbono essere condannati» sono valori morali negativi (Fuller, 1990, p. 148). La ricerca e la scoperta della necessarietà morale nel e attraverso l'immediato significato che abbiamo del nostro io e della struttura della situazione in cui ci troviamo è ciò che chiamiamo coscienza. E una ricerca che mira al «massimo di stabilità, chiarezza e buona organizzazione» (Gurwitsch, 1966, pp. 27-28).

I valori morali possiedono dunque, e a un grado elevato, il carattere di appello al soggetto, piuttosto che derivare da preferenze del soggetto stesso. Certe azioni, dato il loro contesto, presentano chiaramente se stesse come buone o cattive on their on ground e non certamente in un regno interno e

è che il carattere stesso dei passaggi, delle operazioni, dei cambiamenti tra SI e S2 deriva dalla natura dei vettori prodotti in queste difficoltà di struttura per aiutare la situazione, appianarla strutturalmente» (Wertheimer, 1965, p. 255). «Ripeto: la tesi è che proprio le caratteristiche di struttura in SI, con la loro natura particolare, concreta, creano i vettori, con la loro direzione, qualità, intensità; vettori che a loro volta portano ai passaggi e alle operazioni dinamicamente corrispondenti alle esigenze. Questo sviluppo è determinato dal cosiddetto principio della pregnanza, dalle tendenze alla buona forma e dalle varie leggi della forma» (ivi, pp. 255-256). 
privato di giudizi di valore (Fuller, 1990, p. 149). Esse inoltre hanno specifiche conseguenze motivazionali. L'azione morale chiede "di essere eseguita", quella immorale chiede "di trattenersi da essa» (ibid.). D'altro canto, per quanto già ricordato, i valori morali se non possono essere visti come meramente arbitrari, non possono neppure essere considerati come un insieme di regole pronte, a portata di mano.

\section{LA TEORIA DELLE «CAPABILITIES» DI A. SEN E LA TEORIA DEL «FEEDBACK» NEL QUADRO DI UNA VISIONE CONVERSAZIONALE DELL'ATTIVITÀ EDUCATIVA}

Nel 1985 A. Sen ha introdotto il concetto di capability o, al plurale, di capabilities (Sen, 1985) in maniera sistematica collegandolo al concetto di benessere umano (Nussbaum, 2012). Tale concetto è stato ripreso nella sua essenza da M. Nussbaum, anche se con qualche diversa interpretazione sul piano della giustizia sociale di base. Dal nostro punto di vista l'approccio di A. Senn mette in luce come le potenzialità umane sono condizionate nel loro sviluppo dalla realtà esterna con cui interagiscono. Senza l'esistenza effettiva delle condizioni esterne necessarie per uno sviluppo umano potenziale, viene a mancare la libertà fondamentale di realizzarlo. Per questo i due concetti di capability e di sviluppo umano sono considerati come interscambiabili. Il punto centrale sta nel considerare la capability come un combinato di capacità o possibilità di sviluppo interne al soggetto e di opportunità di sviluppo offerte dall'ambiente politico, sociale ed economico (ivi, p. 28) ${ }^{9}$.

L'approccio mette in evidenza il ruolo imprescindibile della realtà esterna con la quale il soggetto interagisce e che lo condiziona nel suo pensare e nel suo agire. Non solo quindi, per usare le espressioni di M. Ferraris, per lui l'ontologia precede l'epistemologia, cioè la conoscenza può svilupparsi solo a partire dal un impatto favorevole con le cose e le persone, ma tale impatto lo condiziona nel suo essere, nel suo divenire e nel suo operare (Ferraris, 2013a). Ciò va attentamente considerato non solo dal punto di vista positivo, ma anche da quello negativo, come vero impedimento o almeno come difficoltà nel crescere e nel fiorire come persona ${ }^{10}$. Sono evidenti le conseguenze sul piano educativo e pedagogico di tale assunto.

9 La Nussbaum, inoltre, precisa: «Storicamente, l'approccio è influenzato da prospettive filosofiche che guardano alla fioritura umana e alla realizzazione di sé dell'uomo da Aristotele a John Stuart Mill in occidente e Rabindranath Tagore in India» (Nussbaum, 2012, p. 30).

10 Il riferimento al «fiorire» della persona è sia dalla Nussbaum, sia da Senn, collegato al concetto aristotelico di eudaimonia. 
Per ricollegarsi all'attuale dibattito italiano ed europeo sull'importanza di promuovere nell'educazione scolastica lo sviluppo di competenze e non solo di saperi, si può considerare il concetto di capability come la possibilità sia interna, sia esterna di raggiungere un adeguato sviluppo della propria capacità di rapportarsi con i compiti da svolgere nella vita sia all'interno della scuola, sia, soprattutto, all'esterno di essa. M. Nussbaum parla esplicitamente di ciò, affermando:

Attualmente, la maggioranza dei paesi, preoccupati per la ricchezza nazionale e desiderosi di acquisire o mantenere una fetta del mercato globale, si concentra sempre più su un numero ristretto di competenze spendibili che si suppone siano generatrici di profitto a breve termine. Le competenze associate alle scienze umane e alle arti - come il pensiero critico, la capacità di immaginare empaticamente la situazione di un'altra persona e una consapevolezza della storia mondiale e dell'attuale ordine economico globale - sono tutte essenziali per una cittadinanza democratica responsabile, oltre che per tutta una serie di altre capacità che le persone possono scegliere di esercitare più tardi nella vita. (Nussbaum, 2012, p. 148)

Nella terminologia di Sen si tratta di raggiungere quei livelli di «funzionamento» della persona agente, che gli consentono di vivere positivamente la sua esistenza di fronte alle sfide che deve affrontare.

\section{LA PROSPETTIVA CONVERSAZIONALE E IL RUOLO DEL «FEEDBACK»}

La prospettiva inizialmente elaborata da A. Sen e ripresa da M. Nussbaum si presta a uno sviluppo particolare che tiene conto del fatto che i processi educativi scolastici si svolgono nel tempo e in un contesto di interazioni tra i docenti e gli studenti, come tra gli studenti stessi e tra i docenti. D. Laurillard (Laurillard, 2012 e 2008) ha sviluppato, a partire dalle ricerche di G. Pask (Pask, 1976) ${ }^{11}$, una lettura dell'attività educativa scolastica di natura conversazionale, che pone al centro della scena la responsabilità progettuale dell'insegnante. Nel contesto dell'attività che si sviluppa in contesti formali dell'apprendimento occorre considerare gli interscambi tra insegnante e studente e tra questi e i suoi compagni di classe. L'insegnante è portatore di conoscenze e di competenze, che, attraverso l'organizzazione di una spazio conversazionale adeguato, tende a promuoverne un analogo sviluppo presso

11 I processi di apprendimento secondo Pask sono basati sui molteplici interscambi comunicativi e collaborativi tra insegnane e allievi al fine di elaborare una interpretazione della realtà. 
i singoli studenti. Il processo che viene messo in atto può essere modellato secondo forme di comunicazione diretta, oppure forme di comunicazione indiretta, che si appoggiano a metafore diverse. Le più diffuse di tali metafore vedono lo studente come uno che può apprendere in vario modo: tramite la ricerca personale o di gruppo, attraverso la discussione comunque sostenuta e guidata dal docente, mediante la produzione di artefatti come testi scritti o prodotti multimediali, collaborando con gli altri nella realizzazione di obiettivi comuni. In questo spazio conversazionale le tecnologie possono, o debbono, svolgere un ruolo fondamentale da molti punti di vista. L'importante è che le attività proposte siano chiaramente dirette al raggiungimento di conoscenze e competenze ritenute fondamentali per la loro crescita. Per questo l'appoggiarsi a forme di allineamento costruttivo delle diverse attività didattiche diventa essenziale (Biggs \& Tang, 2011).

Nella teoria conversazionale di G. Pask gioca un ruolo essenziale il feedback o retroazione che il sistema sul quale si esercita l'azione manifesta. S. Papert osservando i comportamenti degli studenti nella loro attività pratica indica come essi possono apprendere dal feedback, che essi ricevono dai risultati delle loro azioni: «[...] non dal sì-no dall'autorità degli adulti, ma dalla resistenza e dalla guida della realtà. Certe azioni messe in campo non producono i risultati attesi. Altre producono risultati sorprendenti» (Papert, 1996, p. 68). Sulla stessa lunghezza d'onda D. Laurillard insiste sulla distinzione tra feedback intrinseco e feedback estrinseco (Laurillard, 2012, pp. 170174). Il primo tipo di feedback è interno alle azioni stesse nel senso che esso deriva come una conseguenza naturale delle azioni stesse, tenendo conto degli obiettivi intesi. Esso può essere valorizzato dal soggetto per migliorare il suo agire, senza l'intervento istruttivo di altri. Molti degli apprendimenti basati su tentativi ed errori, e sulla riflessione critica del perché di questi ultimi, si fondano proprio su forme di feedback interno. Il secondo tipo di feedback è esterno alle azioni e assume la forma di un commento valutativo su di esse da parte di altri e/o di guide che il soggetto può seguire per raggiungere l'obiettivo desiderato. Si tratta della forma più comune di feedback valorizzato non solo a scuola, ma anche nelle forme di addestramento e di apprendistato. Comunque in ambedue i casi è evidente il ruolo della realtà esterna con la quale ci si deve confrontare per poter conseguire risultati positivi sia nell'apprendere, sia nel realizzare le proprie intenzioni.

Proprio per queste ragioni negli ultimi anni si è avuto uno sviluppo interessante di indagini sul ruolo del feedback nell'ambito educativo e didattico ${ }^{12}$. In particolare si è distinto tra feedback positivo e feedback negativo.

12 Lo studio sulla natura e sugli effetti delle varie forme di feedback ha una lunga storia. I behavioristi tendono ad attribuire al feedback un effetto di rinforzo. Informare il soggetto 
Nel primo caso la situazione può essere così descritta: il docente sollecita un'attività di apprendimento da parte di uno, o più studenti; ad esempio produrre un testo scritto. Il risultato di questa attività può essere approvato dal docente o in toto o in parte, segnalando in quest'ultimo caso possibili miglioramenti. Nel secondo caso il docente non approva quanto realizzato, segnalando errori, carenze, eventualmente manifestando la necessità di riprovare per cercare di ottenere un risultato migliore. Nel processo conversazionale questo nucleo - azione del docente che sollecita un comportamento, azione conseguente dello studente, feedback valutativo da parte del docente - tende a ripetersi in maniera più o meno sistematica in vista del raggiungimento del risultato di apprendimento inteso. In generale si insiste sul migliore valore informativo e formativo del feedback positivo quando si tratta di feedback esterno. Mentre nel caso di un feedback interno sembra svolgere meglio una forma di retro-alimentazione negativa.

Un'analisi sistematica delle ricerche (Thurlins et al., 2013) in merito alle caratteristiche più efficaci dal punto vista didattico del feedback espresso da parte degli insegnanti suggerisce alcune sue caratteristiche fondamentali: sia immediato e correttivo in modo di guidare a elaborare riposte più valide; sia focalizzato sul compito, specifico, dettagliato, chiaro; faccia comprendere quando la risposta può essere corretta; favorisca la comprensione tra l'attuale prestazione e quella desiderata e i percorsi da sviluppare per migliorare; si svolga in un clima di buone relazioni.

\section{Conclusione}

In un suo recente intervento $\mathrm{M}$. Baldacci afferma come da un punto di vista teorico la realtà rappresenti una categoria del discorso pedagogico.

A questo proposito, risulta emblematica la posizione del problematicismo pedagogico. Secondo questa teoria i criteri di scelta educativa sono costituiti dalla fedeltà alla ragione, da una parte, e dall'aderenza alla realtà, dall'altra. Il primo

che una data risposta è corretta gratifica presumibilmente le istanze cognitive affiliative e di affermazione personale. Altre correnti hanno evidenziato gli effetti motivazionali sull'apprendimento successivo e cognitivi. «Esso conferma i significati e le associazioni appropriati, corregge gli errori, chiarisce i fraintendimenti, indica il grado di adeguatezza con cui sono state assimilate parti diverse del compito di apprendimento. Perciò, grazie al feedback ricevuto, aumenta la fiducia nel soggetto della validità dei risultati del suo apprendimento; le sue acquisizioni sono consolidate e gli è più facile mettere a fuoco selettivamente la sua attenzione e suoi sforzi su quegli aspetti del compito che richiedono un ulteriore affinamento». Così M. Pellerey (1983, pp. 149-150), riferendosi ad Ausubel. 
criterio è collegato alla razionalità degli scopi educativi prescelti, il secondo allo stato di cose che caratterizza la situazione educativa e di cui occorre tenere conto nel compiere la scelta. Tale realtà è fatta dall'oggettivizzazione dei processi formativi antecendenti, ma una volta fatta acquisita il carattere di una realtà indipendente dai nostri desideri, e con la quale deve fare i conti la realizzazione dei nostri disegni. (Baldacci, 2013, p. 83)

Come coniugare queste due fedeltà nel concreto dell'azione educativa porta alla necessità di riflettere più in profondità sulle circostanze concrete nelle quali tale azione si svolge. La distinzione di Rawls tra ragione e ragionevolezza può aiutare in tale direzione, evidenziando come la razionalità teoretica svolga un ruolo decisivo nella identificazione del bene da promuovere negli educandi, ma nel contesto dell'impegno educativo concreto diventa indispensabile mettere in gioco proprio quello che abbiamo definito principio di ragionevolezza pedagogica. E ciò evidenzia il ruolo non solo della conoscenza adeguata della realtà di fatto, ma anche del riscontro, o feedback, che deriva dalla messa in opera dei nostri progetti formativi.

Questo ruolo della realtà nel contesto della pratica educativa viene anche sottolineato in maniera molto coinvolgente nella percezione dei valori sia estetici, sia culturali, sia morali da parte dei fondatori della psicologia della Gestalt. Essi hanno insistito sulla sollecitazione che una percezione adeguata della complessità del reale ha sulla formazione dei nostri giudizi di valore $\mathrm{e}$ sullo sviluppo di una quadro di riferimento assiologico. Qualcosa di analogo è stato proposto per quanto riguarda in genere lo sviluppo umano. Questo è legato all'esistenza di effettive capabilities, cioè alla presenza non solo di potenzialità interne di sviluppo, ma soprattutto di condizioni esterne che ne permettano e ne favoriscano il fiorire. In tutti e due gli approcci è essenziale che venga favorito lo sviluppo di un ambiente nel quale il soggetto possa agire e scegliere liberamente se stesso. In qualche modo viene messo in risalto il ruolo di un feedback interno alle azioni stesse basato su processi di natura percettiva e attiva. Tuttavia nell'ambito dell'educazione il ruolo degli altri, siano essi adulti o coetanei, diventa un altro decisivo impatto con la realtà, la quale svolge spesso un'azione di feedback esterno che sollecita, guida e consolida i vari apprendimenti. 


\section{RIFERIMENTI BIBLIOGRAFICI}

Baldacci, M. (2012). Trattato di pedagogia generale. Roma: Carocci.

Baldacci, M. (2013). Il realismo e la ricerca pedagogica. In E. Corbi \& S. Oliverio (a cura di), Realtà tra virgolette? Nuovo realismo e pedagogia. Lecce: Pensa Multimedia.

Berti, E. (1987). Le vie della ragione. Bologna: Il Mulino.

Biggs, J., \& Tang, C. (2011). Teaching for quality learning at university (4th ed.). Buckingham: Society for Research into Higher Education \& Open University Press.

Bongiovanni, G., Sartor, G., \& Valentini, G. (Eds.). (2009). Reasonableness and law. Dordrecht: Springer.

Ferraris, M. (2013a). Realismo positivo. In E. Corbi \& S. Oliverio (a cura di), Realtà tra virgolette? Nuovo realismo e pedagogia (pp. 33-48). Lecce: Pensa Multimedia.

Ferraris, M. (2013b). Realismo positivo. Torino: Rosenberg \& Seller.

Ferraris, M., \& Potworowski, G. (Eds.). (2008). Teaching for wisdom. Dordrecht: Springer.

Fuller, A. R. (1990). Insight into value: An exploration of the premises of a phenomenological psychology. New York: State University of New York.

Gollwitzer, P. M. (1990). Action phases and mind-sets. In E.T. Higgins \& R. M. Sorrentino (Eds.), Motivation \& cognition: Foundations of social behavior, Vol. 2 (pp. 53-92). New York: The Guilford Press.

Gurwitsch, A. (1966). Studies in phenomenology and psychology. Evanston, IL: Northwestern University Press.

Hall, S. S. (2011). Wisdom, from philosophy to neuroscience. New York: Vintage.

Hickeman, T. R. (2004). The reasonableness principle: Reassessing its place in the public sphere. The Cambridge Law Journal, 65(1), 166-189.

Koehler, W. (1938). The place of values in a world of facts. New York: New American Library.

Koehler, W. (1947). Gestalt psychology. New York: New American Library.

Koehler, W. (1967). Some Gestalt problems. In W. D. Ellis (Ed.), Source book of Gestalt psychology (pp. 55-70). New York: Humanities Press.

Koehler, W. (1971). The selected papers of Wolfgang Koehler. New York: Liveright.

La Torre, M., \& Spadaio, A. (2007). La ragionevolezza nel diritto. Torino: Giappichelli, Quaderni del Dipartimento di Scienza e Storia del Diritto.

Laurillard, D. (2008). Digital technologies and their role in achieving our ambitions for education. London: Institute of Education.

Laurillard, D. (2012). Teaching as a design science. Building pedagogical patterns for learning and technology. New York: Routledge. 
Lévinas, E. (1990). Etica e infinito. Milano: Jaca Book.

Maffettone, S. (2010). Introduzione a Rawls. Roma - Bari: Laterza.

Meirieu, P. (1995). La pédagogie entre le faire et le dire. Paris: ESF.

Nussbaum, M. C. (2012). Creare capacità. Liberarsi dalla dittatura del PIL. Bologna: Il Mulino.

Papert, S. (1996). The connected family: Bridging the digital generation gap. Marietta: Longstreet Press.

Pask, G. (1976). Conversation theory: Application in education and epistemology. Amsterdam: Elsevier.

Passaseo, A. M. (2012). La ragionevolezza, capacità da coltivare per il cittadino contemporaneo. Metis, 2. Cfr. http://www.metis.progedit.com/anno-ii-numero2-dicembre-2012/84-saggi/267-la-ragionevolezza-capacita-da-coltivare-peril-cittadino-contemporaneo.html (6 Dicembre 2013).

Pellerey, M. (1983). Verso un sistema di orientamento e formazione a distanza. Quaderni di Formazione ISFOL, Novembre-Dicembre, 149-150.

Pellerey, M. (2013). La ricerca al servizio dell'educazione. Relazione conclusiva. In D. Grządziel (a cura di), La ricerca al servizio dell'educazione (pp. 191-195). Roma: LAS.

Rawls, J. (1993). Political liberalism. New York: Columbia University Press (trad. it., Milano: Edizioni di Comunità, 1994).

Rawls, J. (2006). Giustizia come equità. Una riformulazione. Milano: Feltrinelli.

Rawls, J. (2008). Una teoria della giustizia. Milano: Feltrinelli.

Sandulli, M. (1975). Il principio di ragionevolezza nella giurisprudenza costituzionale. Diritto e Società, 565.

Schwartz, B. (2011). Practical wisdom. London: Penguin.

Sen, A. (1985). Commodities and capabilities. Amsterdam: North Holland.

Sen, A. (1993). Capability and well-being. In M. Nussbaum \& A. Sen (Eds.), The quality of life (pp. 30-53). New York: Oxford Clarendon Press.

Spadaro, A. (2007). La ragionevolezza nel diritto. Torino: Giappichelli, Quaderni del Dipartimento di Scienza e Storia del Diritto.

Sternberg, R., \& Jordan, J. (2005). A handbook of wisdom. New York: Cambridge University Press.

Thurlins, M., Vermeulen, M., Bastiaen, T., \& Stijnen, S. (2013). Understanding feedback: A learning theory perspective. Educational Research Review, 9, 1-15.

Viola, F. (2003). La ragionevolezza positiva secondo Rawls. In C. Vigna (a cura di), Etiche e politiche della post-modernità (pp. 163-181). Milano: Vita e Pensiero.

Wertheimer, M. (1961). Some problems in the theory of ethics. In M. Henle (Ed.), Documents of Gestalt psychology (pp. 29-41). Berkeley, CA: University of California Press. 
Wertheimer, M. (1965). Il pensiero produttivo. Firenze: Editrice Universitaria.

Wertheimer, M. (1967). The general theoretical situation. In W. D. Ellis (Ed.), A source book of Gestalt psychology (pp. 71-88). New York: Humanities Press.

Zagrebelsky, G. (1983). La giustizia costituzionale. Bologna: Il Mulino.

\section{Riassunto}

Il contributo intende approfondire il ruolo che nell'azione educativa deriva svolge l'impatto con la realtà e in particolare il dover tener conto del "volto" dell'altro, soprattutto quando egli resiste di fronte ai nostri propositi e l'educatore deve assumere la responsabilità etica che ne deriva. A questo fine viene esplorato l'apporto che, a partire da alcune suggestioni provenienti da J. Rawls, può derivare dalla considerazione di quello che nell'ambito della giustizia è stato definito il canone o principio di ragionevolezza. Quindi, si presenta la posizione degli psicologi della "Gestalt» in merito alla sollecitazione che deriva dal cogliere la sollecitazione che proviene dalla realtà nello sviluppo dei valori estetici, culturali e morali e alle conseguenze educative che ne derivano. Sulla base della teoria delle "capabilities» di A. Sen e M. Nussbaum, viene, infine, evidenziata l'importanza di valorizzare in ambito pedagogico la prospettiva conversazionale avviata da $G$. Pask e ripresa di D. Laurillard e il ruolo che il "feedback» sia negativo, sia positivo assume. Si conclude sottolineando come nell'ambito dell'agire educativo l'incontro con l'altro può costituire un decisivo impatto con una realtà che è sorgente di stimolo, orientamento e guida all'azione.

Parole chiave: Educazione, Feedback, Ragionevolezza, Realtà, Valori. 